\title{
Padrões das síndromes de dispersão de plantas em áreas com diferentes graus de pluviosidade, PE, Brasil
}

\author{
Maria Carolina Nunes Alves da Silva ${ }^{1,2}$ e Maria Jesus Nogueira Rodal ${ }^{1}$
}

Recebido em 06/06/2008. Aceito em 27/02/2009

RESUMO - (Padrões das síndromes de dispersão de plantas em áreas com diferentes graus de pluviosidade, PE, Brasil). Este trabalho objetivou descrever as proporções de síndromes de dispersão em três remanescentes de vegetação nativa com distintos totais pluviométricos e número de meses secos. Durante três anos, foram coletados mensalmente frutos e diásporos de 107 espécies, os quais foram analisados e descritos. As áreas com precipitação inferior a 700 $\mathrm{mm} / \mathrm{ano}$ (Floresta e Caruaru) assemelham-se em todas as síndromes de dispersão analisadas (autocoria, anemocoria e zoocoria), enquanto que Tapacurá (precipitação média anual de $1299 \mathrm{~mm}$ ) distancia-se principalmente pela zoocoria, que variou de $29 \%$ (Floresta) a $51 \%$ (Tapacurá). Em termos de estratificação vertical das síndromes de dispersão, houve maior proporção de zoocoria no sub-bosque de Tapacurá, enquanto que nas áreas mais secas (Floresta e Caruaru) a autocoria e anemocoria foram representativas no dossel. Este trabalho mostra que, associados à diminuição da pluviosidade, os vetores abióticos tornam-se mais importantes e que as proporções das síndromes de dispersão variam de acordo com a estratificação.

Palavras-chave: Caatinga, estratificação, floresta estacional, sazonalidade

ABSTRACT - (Patterns of dispersal syndromes of plants in areas with different total rainfall, Pernambuco state, Brazil). This study aimed to describe the proportion of dispersal syndromes in three remnants of native vegetation with distinct total rainfall and number of dry months. During three years of monthly sampling we collected and described fruits and diaspores of 107 species. The areas with precipitation less than $700 \mathrm{~mm} / \mathrm{year}$ (Floresta and Caruaru) showed similar proportions of dispersal syndromes (autochory, anemochory and zoochory), whereas Tapacurá (mean annual precipitation 1300 $\mathrm{mm}$ ) was distinguished mainly by zoochory, which varied from $29 \%$ (Floresta) to $51 \%$ (Tapacurá) of the species. The distribution of dispersal syndromes along a vertical gradient showed a higher proportion of zoochory in the understory of more humid areas such as Tapacurá, while in drier areas (Floresta and Caruaru) autochory and anemochory were more representative in the canopy. These results indicate that the abiotic vectors become more important with the decrease in rainfall, and also that dispersal-syndrome proportions differ with stratification.

Key words: Caatinga, seasonal forest, seasonality, stratification

\section{Introdução}

O efeito da sazonalidade nos padrões de síndromes de dispersão de comunidades vegetais tropicais vem sendo estudado desde a década de 70 (Frankie et al. 1974, Gentry 1983). Supõe-se que nos ecossistemas com pluviosidade elevada e bem distribuída ao longo do ano, ocorre predomínio de plantas dispersas por vertebrados e, à medida que aumenta o grau de sazonalidade e diminui a precipitação, os ambientes tornam-se mais secos e os vetores abióticos, tais como o vento e a gravidade, vão ganhando importância (Griz et al. 2002, Vicente et al. 2003). Como exemplo, podemos citar o estudo de Justiniano \& Fredericksen (2000) em formações florestais secas da Bolívia e o de Mantovani \& Martins (1988) no cerrado brasileiro, os quais revelaram que as plantas tendem a dispersar seus diásporos por meios abióticos, envolvendo autocoria e anemocoria, assim como o estudo de Griz \& Machado (1998), em um remanescente de Mata Atlântica, onde a síndrome preponderante foi a zoocoria. É possível que a razão para haver um maior número de agentes bióticos em ambientes mais úmidos esteja relacionada com o fato das florestas úmidas apresentarem maior riqueza de animais e plantas arbóreas (Pianka 1982 Gentry 1983). De fato, nas florestas tropicais, de 50 a $90 \%$ das árvores e arbustos apresentam síndrome zoocórica, sendo a maioria da biomassa dos vertebrados mantida por frutos carnosos (Fleming 1979; Howe \& Smallwood 1982). Além disso, nos ecossistemas úmidos, devido à proteção das folhagens, os frutos carnosos se mantêm viáveis por mais tempo, favorecendo a dispersão zoocórica (Weiser \& Godoy 2001).
Estudos que investigam a variação dos mecanismos de dispersão ao longo dos diferentes estratos verticais no nível de comunidades são escassos, pois a maioria se detém a observar um único mecanismo, espécie, ou outros fatores locais e influências (Drezner et al. 2001 Rey et al. 2002). Wikander (1984) relatou que, em uma floresta decídua na Venezuela, a anemocoria foi a síndrome mais relevante, principalmente no estrato superior, e a zoocoria no inferior. Padrão semelhante foi revelado por Justiniano \& Fredericksen (2000), em florestas secas, com espécies zoocóricas e autocóricas melhor representadas no estrato inferior. Espécies emergentes e trepadeiras são geralmente anemocóricas, sendo este tipo de dispersão freqüente entre espécies que são relativamente altas dentro de seus respectivos habitats, pois, a velocidade do vento no sub-bosque é menor não favorecendo este tipo de dispersão (Morellato \& Leitão-Filho 1996, Willson \& Traveset 2000).

Apesar do consenso de que entender o funcionamento de sistemas tropicais é fundamental para seu manejo, formações sazonalmente secas são comparativamente menos estudadas que as úmidas (Mooney et al. 1995). No caso da Caatinga do nordeste brasileiro, os primeiros estudos funcionais enfocaram a fenologia (Barbosa et al. 1989, Machado et al. 1997), as síndromes de dispersão (Griz \& Machado 2001, Barbosa et al. 2002, Griz et al. 2002, Tabarelli et al. 2003, Vicente et al. 2003), os sistemas de reprodução (Machado \& Lopes 2004) e o banco de sementes (Costa \& Araújo 2003). A respeito das florestas estacionais da região, a maioria dos estudos trata de aspectos fitogeográficos (para revisão ver Rodal \& Sales 2007).

\footnotetext{
1 Universidade Federal Rural de Pernambuco, Departamento de Biologia, Área de Botânica, Laboratório de Fitossociologia, Recife, PE, Brasil

2 Autora para correspondência: c.nunes@ibest.com.br
} 
Este trabalho, realizado em remanescentes de vegetação nativa em Pernambuco, objetiva levantar evidências de que, à medida que aumenta a pluviosidade e declina a sazonalidade, os vetores bióticos tornam-se mais importantes, e que as proporções das síndromes de dispersão variam de acordo com a estratificação. Neste sentido, espera-se que, em áreas com acentuada sazonalidade climática e baixa precipitação, como a Caatinga, haja um predomínio de espécies autocóricas e anemocóricas e que, em formações florestais com um clima menos sazonal e maiores totais pluviométricos, predominem vetores bióticos; espera-se também que as proporções das síndromes variem com a estrutura vertical da floresta.

\section{Material e métodos}

Área de estudo - As coletas foram realizadas em três áreas do estado de Pernambuco: duas em áreas com vegetação de Caatinga, com diferentes fisionomias, e uma em remanescente de Mata Atlântica. A primeira área fica situada na Reserva Particular do Patrimônio Natural Maurício Dantas (RPPN), no município de Floresta $\left(08^{\circ} 18^{\prime} 45^{\prime \prime} \mathrm{S}, 30^{\circ} 11^{\prime} 43^{\prime \prime} \mathrm{W}\right)$. A precipitação média anual é da ordem de $503 \mathrm{~mm}$, com 11 meses abaixo dos $100 \mathrm{~mm}$ (LAMEPE 2009) (Fig. 1). A vegetação nesta área é classificada por Veloso et al. (1991) como savana-estépica e apresenta fisionomia arbustivo-arbórea. A segunda área está localizada na estação experimental da Empresa Pernambucana de Pesquisa Agropecuária (IPA), no município de Caruaru (8¹4'18"S, $35^{\circ} 55^{\prime} 20^{\prime \prime} \mathrm{W}$ ). A pluviosidade média anual é de $662 \mathrm{~mm}$, havendo precipitação abaixo dos $100 \mathrm{~mm}$ em todos os meses (dados do posto da estação experimental do IPA) (figura 1). É classificada por Veloso et al. (1991) como savana-estépica e apresenta fisionomia arbórea. A terceira área situa-se na Estação Ecológica do Tapacurá (Tapacurá), no município de São Lourenço da Mata $\left(08^{\circ} 01^{\prime} \mathrm{S}, 35^{\circ} 11^{\prime} \mathrm{W}\right)$, e consiste de um remanescente de floresta estacional decidual de terras baixas (sensu Veloso et al. 1991). A precipitação média anual é de $1.299 \mathrm{~mm}$, com seis meses abaixo dos $100 \mathrm{~mm}$ (dados do Posto e Barragem de Tapacurá, do Departamento de Hidrometeorologia da Secretaria de Recursos Hídricos de Pernambuco) (Fig. 1).

Coleta e tratamento de dados - Durante três anos, foram realizadas excursões mensais para coleta de frutos e sementes nas parcelas, ou nas proximidades, dos seguintes levantamentos quantitativos: Rodal et al. (2008), na RPPN, Floresta; Alcoforado-Filho et al. (2003), no IPA, Caruaru; e Andrade \& Rodal (2004), em Tapacurá, São Lourenço da Mata. As espécies selecionadas em cada uma das três áreas foram aquelas definidas entre as de maior importância estrutural nos levantamentos acima citados. Os frutos/ sementes das espécies lenhosas e cactáceas foram fixados em formol-ácido acético-álcool a 50\%, havendo ainda o registro fotográfico e coleta de flores das espécies. As espécies não coletadas em fruto tiveram o material examinado a partir de exsicatas tombadas no herbário Professor Vasconcelos Sobrinho. As espécies foram organizadas em famílias de acordo com o sistema de Cronquist (1981), exceto para Leguminosae, que seguiu Pohill et al. (1981). O tipo de fruto foi definido conforme Spjut (1994). As síndromes de dispersão foram classificadas segundo Van der Pijl (1982). Foi realizada uma análise de correlação de Spearman (Ayres et al. 2000) entre os valores de precipitação mensal e o número de espécies de dossel e sub-bosque com as diferentes síndromes de dispersão das três áreas aqui estudadas e com os dados dos trabalhos realizados por Machado et al. (1997) e Griz \& Machado (2001). No caso dos dois últimos estudos, procedeu-se à classificação das espécies como de dossel ou de sub-bosque.

\section{Resultados}

Área Floresta - Das 24 espécies descritas, 17 tiveram frutos secos (Tab. 1). Daquele total, 14 espécies foram definidas como pertencentes ao dossel e dez ao sub-bosque. Foram observados três tipos de síndromes de dispersão: a autocoria (incluindo a barocoria) com 54\% das espécies, seguida da zoocoria (29\%) e anemocoria (17\%).
Todas as 13 espécies autocóricas tiveram frutos secos, a exemplo de Anadenanthera colubrina, Bauhinia cheilantha, Mimosa ophthalmocentra e Senna macranthera. Essas 13 espécies estão distribuídas tanto no dossel (8) quanto no sub-bosque (5). As espécies zoocóricas apresentaram frutos carnosos, a exemplo de Arrojadoa rhodantha e Commiphora leptophloeos, ocorrendo tanto no dossel (2) como no subbosque (5). As quatro espécies anemocóricas tiveram frutos secos, e todas são características do dossel, como Amburana cearensis, Aspidosperma pyrifolium, Myracrodruon urundeuva e Schinopsis brasiliensis.

Área Caruaru - Das 33 espécies descritas, 20 apresentaram frutos secos (Tab. 1). Do total de espécies, 14 foram definidas como pertencentes ao sub-bosque (por exemplo: Maytenus rigida, Acacia paniculata e Eugenia uvalha) e 19 ao dossel (exemplos: Chorisia glaziovii e Ziziphus joazeiro). Foram observados três tipos de síndromes de dispersão: a autocoria (incluindo a barocoria) com $54,5 \%$ das espécies, seguida da zoocoria $(33,4 \%)$ e anemocoria $(12,1 \%)$.

Nas 18 espécies autocóricas, houve maioria de frutos secos, como Acacia glomerosa, Croton blanchetianus e Caesalpinia pyramidalis. Essas 18 espécies estão distribuídas tanto no dossel (10) quanto no sub-bosque (8). As espécies zoocóricas apresentaram em sua totalidade frutos carnosos, a exemplo de Allophylus quercifolius e Cordia globosa, presentes no sub-bosque. As quatro espécies anemocóricas tiveram frutos secos (Cedrela odorata, Coutarea hexandra, Myracrodruon urundeuva e Schinopsis brasiliensis), todas típicas do dossel, à exceção de $C$. hexandra.

Área Tapacurá - Das 62 espécies descritas, 36 apresentaram frutos carnosos (Tab. 1). Daquele total, 32 espécies foram definidas como pertencentes ao sub-bosque (exemplos: Cordia superba e Maytenus $\mathrm{cf}$. distichophylla) e 30 ao dossel (exemplos: Albizia polycephala e Luehea paniculata). Foram observados três tipos de síndromes de dispersão; a zoocoria foi o modo de dispersão mais representado (51,6\% das espécies), seguido da autocoria (incluindo a barocoria) $(38,7 \%)$ e anemocoria $(9,7 \%)$.

As espécies zoocóricas apresentaram frutos carnosos, a exemplo de Casearia sylvestris, Psidium araca, típicas do sub-bosque, e Pouteria glomerata característica do dossel. Nas 24 espécies autocóricas, houve maioria de frutos secos, a exemplo de, Caesalpinia echinata, Chamaecrista ensiformis e Christiana africana, todas típicas do dossel. As seis espécies anemocóricas apresentaram frutos secos, como Alseis pickelii e Luehea paniculata, características do dossel.

Comparação entre as áreas - Os resultados das três áreas de estudo, apontam para uma diminuição no número de frutos secos à medida que a sazonalidade torna-se menor, ocorrendo o inverso com os frutos carnosos (Fig. 2).

Relacionando a estratificação vertical com os modos de dispersão, nota-se que, no dossel, a a utocoria diminuiu de Floresta para Tapacurá (Fig. 3). O número de espécies zoocóricas no sub-bosque aumentou ligeiramente de Floresta para Caruaru, e acentuadamente de Caruaru para Tapacurá, 
Tabela 1. Atributos das espécies estudadas nas áreas de Floresta (FLO), Caruaru (CAR) e Tapacurá (TAP), PE, Brasil. EV $=$ estrato vertical, DO = Dossel, SB = sub-bosque, $\mathrm{X}=$ presença da espécie na área, - = ausência da espécie na área, $\mathrm{SD}=$ Síndrome de dispersão, $\mathrm{ANE}=\mathrm{Anemocoria}, \mathrm{ZOO}=\mathrm{Zoocoria}, \mathrm{AUT}=$ Autocoria, $\mathrm{CO}=$ Consistência, $\mathrm{Seco}=\mathrm{S}$, Carnoso $=\mathrm{C}, \mathrm{DE}=$ Deiscência, $\mathrm{I}=$ Indeiscente, $\mathrm{D}=$ Deiscente, $*$ = material descrito a partir de exsicatas depositadas no herbário professor Vasconcelos Sobrinho

\begin{tabular}{|c|c|c|c|c|c|c|c|c|c|}
\hline \multirow[b]{2}{*}{ Família / espécie } & \multicolumn{5}{|c|}{ Ocorrência } & \multirow[b]{2}{*}{$\mathrm{CO}$} & \multirow[b]{2}{*}{ DE } & \multirow[b]{2}{*}{ Coloração } & \multirow[b]{2}{*}{ Tipos de fruto } \\
\hline & $\mathbf{E V}$ & FLO & CAR & TAP & SD & & & & \\
\hline \multicolumn{10}{|l|}{ Anacardiaceae } \\
\hline Myracrodruon urundeuva Allemão & DO & $\mathrm{X}$ & $\mathrm{X}$ & - & ANE & $\mathrm{S}$ & I & vináceo & drupa \\
\hline Schinopsis brasiliensis Engl. & DO & $\mathrm{X}$ & $\mathrm{X}$ & - & ANE & $\mathrm{S}$ & I & marrom & sâmara \\
\hline Spondias lutea Royen ex Blume & DO & - & - & $\mathrm{X}$ & $\mathrm{ZOO}$ & $\mathrm{C}$ & I & laranja & drupa \\
\hline \multicolumn{10}{|l|}{ Apocynaceae } \\
\hline Aspidosperma pyrifolium Mart. & DO & $\mathrm{X}$ & - & - & ANE & $\mathrm{S}$ & $\mathrm{D}$ & castanho & folículo \\
\hline \multicolumn{10}{|l|}{ Bombacaceae } \\
\hline Chorisia glaziovii (O. Kuntze) E. Santos & DO & - & $\mathrm{X}$ & - & AUT & S & $\mathrm{D}$ & marrom & cápsula \\
\hline Eriotheca crenulaticalyx A. Robyns* & DO & - & - & $\mathrm{X}$ & ANE & $\mathrm{S}$ & $\mathrm{D}$ & castanho & cápsula \\
\hline \multicolumn{10}{|l|}{ Boraginaceae } \\
\hline Cordia globosa (Jacq.) Kunth & SB & - & $\mathrm{X}$ & - & $\mathrm{ZOO}$ & $\mathrm{C}$ & I & vermelho & drupa \\
\hline Cordia superba Cham. & $\mathrm{SB}$ & - & - & $\mathrm{X}$ & $\mathrm{ZOO}$ & $\mathrm{C}$ & I & branco & drupa \\
\hline \multicolumn{10}{|l|}{ Burseraceae } \\
\hline Commiphora leptophloeos (Mart.) J.B. Gillett. & DO & $\mathrm{X}$ & $\mathrm{X}$ & - & $\mathrm{ZOO}$ & $\mathrm{C}$ & $\mathrm{D}$ & verde-vináceo & drupa \\
\hline Protium heptaphyllum (Aubl.) Marchand & $\mathrm{SB}$ & - & - & $\mathrm{X}$ & ZOO & $\mathrm{C}$ & $\mathrm{D}$ & rosa & drupa \\
\hline \multicolumn{10}{|l|}{ Cactaceae } \\
\hline Arrojadoa rhodantha (Gürke) Britton \& Rose & SB & $\mathrm{X}$ & - & - & $\mathrm{ZOO}$ & $\mathrm{C}$ & I & vináceo & baga \\
\hline Cereus jamacaru DC. & DO & - & $\mathrm{X}$ & - & $\mathrm{ZOO}$ & $\mathrm{C}$ & $\mathrm{I}$ & vermelho-vináceo & baga \\
\hline Cereus jamacaru DC. & SB & $\mathrm{X}$ & - & - & $\mathrm{ZOO}$ & $\mathrm{C}$ & I & vermelho-vináceo & baga \\
\hline Harrisia adscendens (Gürke) Britton \& Rose & $\mathrm{SB}$ & $\mathrm{X}$ & - & - & $\mathrm{ZOO}$ & $\mathrm{C}$ & I & vermelho-vináceo & baga \\
\hline Pilosocereus gounellei Byles \& G.D.Rowley & SB & $\mathrm{X}$ & - & - & $\mathrm{ZOO}$ & $\mathrm{C}$ & I & verde & baga \\
\hline Pilosocereus pachycladus Zappi. & DO & - & $\mathrm{X}$ & - & $\mathrm{ZOO}$ & $\mathrm{C}$ & I & amarelo & baga \\
\hline Opuntia brasiliensis (Willd.) Haw. & DO & - & $\mathrm{X}$ & - & $\mathrm{ZOO}$ & $\mathrm{C}$ & I & vináceo & baga \\
\hline Tacinga palmadora Britton \& Rose & $\mathrm{SB}$ & $\mathrm{X}$ & - & - & $\mathrm{ZOO}$ & $\mathrm{C}$ & I & vináceo & baga \\
\hline \multicolumn{10}{|l|}{ Capparaceae } \\
\hline Capparis flexuosa L. & DO & $\mathrm{X}$ & $\mathrm{X}$ & $\mathrm{X}$ & $\mathrm{ZOO}$ & $\mathrm{C}$ & $\mathrm{D}$ & vináceo & cápsula \\
\hline Capparis jacobinae Moric. & DO & - & $\mathrm{X}$ & - & AUT & $\mathrm{C}$ & I & verde & baga \\
\hline Capparis nectaria Vell. & DO & - & - & $\mathrm{X}$ & AUT & $\mathrm{C}$ & I & verde & baga \\
\hline \multicolumn{10}{|l|}{ Celastraceae } \\
\hline Maytenus cf. distichophylla Mart. & SB & - & - & $\mathrm{X}$ & $\mathrm{ZOO}$ & $\mathrm{C}$ & $\mathrm{D}$ & vermelho & drupa \\
\hline Maytenus rigida Mart.* & SB & - & $\mathrm{X}$ & - & $\mathrm{ZOO}$ & $\mathrm{C}$ & $\mathrm{D}$ & marrom-vináceo & cápsula \\
\hline \multicolumn{10}{|l|}{ Connaraceae } \\
\hline Rourea sp. & SB & - & - & $\mathrm{X}$ & $\mathrm{ZOO}$ & $\mathrm{C}$ & I & amarelo & drupa \\
\hline \multicolumn{10}{|l|}{ Euphorbiaceae } \\
\hline Actinostemon concolor (Spreng.) Müll. Arg. & $\mathrm{SB}$ & - & - & $\mathrm{X}$ & AUT & $\mathrm{S}$ & $\mathrm{D}$ & verde & cocarium \\
\hline Cnidoscolus bahianus (Ule) Pax \& K.Hoffm. & DO & $\mathrm{X}$ & - & - & AUT & $\mathrm{S}$ & $\mathrm{D}$ & verde & cocarium \\
\hline Cnidoscolus quercifolius Pohl. & DO & $\mathrm{X}$ & - & - & AUT & $\mathrm{S}$ & $\mathrm{D}$ & verde & cocarium \\
\hline Croton blanchetianus Baill & $\mathrm{SB}$ & $\mathrm{X}$ & $\mathrm{X}$ & - & AUT & $\mathrm{S}$ & $\mathrm{D}$ & verde & cocarium \\
\hline Croton rhamnifolioides Pax \& K.Hoffm. & SB & $\mathrm{X}$ & $\mathrm{X}$ & - & AUT & $\mathrm{S}$ & $\mathrm{D}$ & verde & cocarium \\
\hline Jatropha mollissima Pohl \& Baill. & DO & $\mathrm{X}$ & $\mathrm{X}$ & - & AUT & S & $\mathrm{D}$ & marrom & cocarium \\
\hline Manihot dichotoma Ule & DO & - & $\mathrm{X}$ & - & AUT & S & $\mathrm{D}$ & marrom & cocarium \\
\hline Manihot cf. epruinosa Pax \& K.Hoffm. & DO & $\mathrm{X}$ & - & - & AUT & $\mathrm{S}$ & $\mathrm{D}$ & marrom & cocarium \\
\hline Maprounea guianensis Aubl.* & DO & - & $\mathrm{X}$ & - & AUT & $\mathrm{S}$ & $\mathrm{D}$ & vinácea & cocarium \\
\hline Richeria cf. grandis Vahl. & $\mathrm{SB}$ & - & - & $\mathrm{X}$ & AUT & S & $\mathrm{D}$ & verde & cocarium \\
\hline Sapium lanceolatum (Müll. Arg.) Herber & DO & - & $\mathrm{X}$ & - & AUT & $\mathrm{S}$ & $\mathrm{D}$ & verde & cápsula \\
\hline Euphorbiaceae A & $\mathrm{SB}$ & - & - & $\mathrm{X}$ & AUT & $\mathrm{S}$ & $\mathrm{D}$ & verde & cocarium \\
\hline Euphorbiaceae B & SB & - & - & $\mathrm{X}$ & AUT & S & $\mathrm{D}$ & verde & cocarium \\
\hline \multicolumn{10}{|l|}{ Flacourtiaceae } \\
\hline Casearia sylvestris $\mathrm{Sw} . *$ & SB & - & - & $\mathrm{X}$ & $\mathrm{ZOO}$ & $\mathrm{C}$ & I & marrom & baga \\
\hline Lauraceae & & & & & & & & & \\
\hline Ocotea bracteosa (Meisn.) Mez* & DO & - & - & $\mathrm{X}$ & AUT & $\mathrm{C}$ & I & marrom & drupa \\
\hline Ocotea gardneri (Meisn.) Mez* & DO & - & - & $\mathrm{X}$ & AUT & $\mathrm{C}$ & I & marrom & drupa \\
\hline Lauraceae A & $\mathrm{SB}$ & - & - & $\mathrm{X}$ & AUT & $\mathrm{S}$ & $\mathrm{I}$ & verde & drupa \\
\hline
\end{tabular}


Tabela 1 (Continuação).

\begin{tabular}{|c|c|c|c|c|c|c|c|c|c|}
\hline \multirow[b]{2}{*}{ Família / espécie } & \multicolumn{5}{|c|}{ Ocorrência } & \multirow[b]{2}{*}{$\mathrm{CO}$} & \multirow[b]{2}{*}{ DE } & \multirow[b]{2}{*}{ Coloração } & \multirow[b]{2}{*}{ Tipos de fruto } \\
\hline & $\mathbf{E V}$ & FLO & CAR & TAP & SD & & & & \\
\hline \multicolumn{10}{|l|}{ Lecythidaceae } \\
\hline Eschweilera ovata (Cambess.) Miers & DO & - & - & $\mathrm{X}$ & AUT & $\mathrm{S}$ & $\mathrm{D}$ & verde & baga \\
\hline Gustavia augusta $\mathrm{L}$. & SB & - & - & $\mathrm{X}$ & AUT & S & $\mathrm{D}$ & vináceo & baga \\
\hline Lecythis sp. & DO & - & - & $\mathrm{X}$ & AUT & S & $\mathrm{D}$ & marrom & baga \\
\hline \multicolumn{10}{|l|}{ Leguminosae } \\
\hline Acacia glomerosa Benth. & SB & - & $\mathrm{X}$ & - & AUT & $\mathrm{S}$ & $\mathrm{D}$ & marrom-escuro & legume \\
\hline Acacia paniculata Willd.* & SB & - & $\mathrm{X}$ & - & AUT & S & $\mathrm{D}$ & marrom & legume \\
\hline Albizia polycephala (Benth.) Killip. & DO & - & - & $\mathrm{X}$ & AUT & S & $\mathrm{D}$ & marrom-claro & legume \\
\hline Amburana cearensis (Allemão) A.C.Sm. & DO & $\mathrm{X}$ & - & - & ANE & S & $\mathrm{D}$ & marrom-vináceo & criptossâmara \\
\hline Anadenanthera colubrina (Vell.) Brenam & DO & $\mathrm{X}$ & $\mathrm{X}$ & - & AUT & S & $\mathrm{D}$ & marrom-vináceo & legume \\
\hline Bauhinia cheilantha (Bong.) Steud. & SB & $\mathrm{X}$ & $\mathrm{X}$ & - & AUT & S & $\mathrm{D}$ & marrom-vináceo & legume \\
\hline Bauhinia sp. & SB & - & - & $\mathrm{X}$ & AUT & S & $\mathrm{D}$ & marrom & legume \\
\hline Bowdichia virgilioides Kunth* & DO & - & - & $\mathrm{X}$ & AUT & S & I & amarelo & legume \\
\hline Caesalpinia echinata Lam. & DO & - & - & $\mathrm{X}$ & AUT & S & $\mathrm{D}$ & marrom & legume \\
\hline Caesalpinia gardneriana Benth. & DO & $\mathrm{X}$ & - & - & AUT & S & $\mathrm{D}$ & marrom & legume \\
\hline Caesalpinia pyramidalis Tul. & DO & - & $\mathrm{X}$ & - & AUT & S & $\mathrm{D}$ & marrom-claro & legume \\
\hline Chamaecrista ensiformis (Vell.) H.S.Irwin \& Barneby* & DO & - & - & $\mathrm{X}$ & AUT & S & $\mathrm{D}$ & marrom & legume \\
\hline Dialium guianense (Aubl.) Sandwith & DO & - & - & $\mathrm{X}$ & AUT & S & I & marrom-vináceo & drupa \\
\hline Mimosa malacocentra Mart. & DO & - & $\mathrm{X}$ & - & AUT & S & I & marrom-escuro & craspédio \\
\hline Mimosa ophthalmocentra Mart. ex Benth. & SB & $\mathrm{X}$ & - & - & AUT & S & I & marrom & craspédio \\
\hline Mimosa tenuiflora (Willd.) Poir.* & DO & $\mathrm{X}$ & - & - & AUT & S & $\mathrm{D}$ & marrom-claro & legume \\
\hline Piptadenia stipulacea (Benth.) Ducke & DO & $\mathrm{X}$ & $\mathrm{X}$ & - & AUT & S & $\mathrm{D}$ & marrom-claro & legume \\
\hline Pithecellobium tortum Mart. & SB & - & $\mathrm{X}$ & - & AUT & S & $\mathrm{D}$ & marrom & legume \\
\hline Plathymenia foliolosa Benth. & DO & - & - & $\mathrm{X}$ & ANE & S & $\mathrm{D}$ & marrom-escuro & legume \\
\hline Pterocarpus rohrii Vahl.* & DO & - & - & $\mathrm{X}$ & ANE & S & I & marrom & sâmara \\
\hline Senna macranthera H.S.Irwin \& Barneby & SB & $\mathrm{X}$ & - & - & AUT & S & $\mathrm{D}$ & marrom & legume \\
\hline Senna sp. & SB & - & $\mathrm{X}$ & - & AUT & $\mathrm{S}$ & $\mathrm{D}$ & marrom & legume \\
\hline Swartzia pickelii Killip ex Ducke & DO & - & - & $\mathrm{X}$ & AUT & S & I & amarelo & legume \\
\hline \multicolumn{10}{|l|}{ Malpighiaceae } \\
\hline Byrsonima sericea DC. & DO & - & - & $\mathrm{X}$ & $\mathrm{ZOO}$ & $\mathrm{C}$ & I & verde & drupa \\
\hline \multicolumn{10}{|l|}{ Melastomataceae } \\
\hline Miconia albicans (Sw.) Triana & SB & - & - & $\mathrm{X}$ & $\mathrm{ZOO}$ & $\mathrm{C}$ & I & bege & baga \\
\hline Miconia prasina $(\mathrm{Sw}.) \mathrm{DC}$. & SB & - & - & $\mathrm{X}$ & $\mathrm{ZOO}$ & $\mathrm{C}$ & I & marrom & baga \\
\hline \multicolumn{10}{|l|}{ Meliaceae } \\
\hline Cedrela odorata $\mathrm{L}$. & DO & - & $\mathrm{x}$ & - & ANE & S & $\mathrm{D}$ & marrom & cápsula \\
\hline \multicolumn{10}{|l|}{ Moraceae } \\
\hline Sorocea hilarii Gaudich. & SB & - & - & $\mathrm{X}$ & $\mathrm{ZOO}$ & $\mathrm{C}$ & I & verde & drupa \\
\hline \multicolumn{10}{|l|}{ Myristicaceae } \\
\hline Virola gardneri (DC.) Mart. & DO & - & - & $\mathrm{X}$ & $\mathrm{ZOO}$ & $\mathrm{C}$ & $\mathrm{D}$ & marrom & cocum \\
\hline \multicolumn{10}{|l|}{ Myrtaceae } \\
\hline Campomanesia dichotoma (O. Berg) Mattos & SB & - & - & $\mathrm{X}$ & $\mathrm{ZOO}$ & $\mathrm{C}$ & I & lilás & baga \\
\hline Eugenia uvalha Camb. & SB & - & $\mathrm{X}$ & - & $\mathrm{ZOO}$ & $\mathrm{C}$ & I & vermelho & drupa \\
\hline Myrcia fallax (Rich.) DC.* & SB & - & - & $\mathrm{X}$ & $\mathrm{ZOO}$ & $\mathrm{C}$ & I & verde & drupa \\
\hline Psidium araca Raddi & SB & - & - & $\mathrm{X}$ & $\mathrm{ZOO}$ & $\mathrm{C}$ & I & marrom & baga \\
\hline Myrtaceae A & SB & - & - & $\mathrm{X}$ & $\mathrm{ZOO}$ & $\mathrm{C}$ & I & marrom & drupa \\
\hline Myrtaceae B & SB & - & - & $\mathrm{X}$ & $\mathrm{ZOO}$ & $\mathrm{C}$ & I & vináceo & drupa \\
\hline Myrtaceae C & SB & - & - & $\mathrm{X}$ & $\mathrm{ZOO}$ & $\mathrm{C}$ & I & verde & drupa \\
\hline Myrtaceae D & SB & - & - & $\mathrm{X}$ & $\mathrm{ZOO}$ & $\mathrm{C}$ & I & vináceo & drupa \\
\hline Myrtaceae E & SB & - & - & $\mathrm{X}$ & $\mathrm{ZOO}$ & $\mathrm{C}$ & I & verde & drupa \\
\hline Myrtaceae F & SB & - & - & $\mathrm{X}$ & $\mathrm{ZOO}$ & $\mathrm{C}$ & I & vináceo & drupa \\
\hline \multicolumn{10}{|l|}{ Polygonaceae } \\
\hline Coccoloba mollis Casar.* & DO & - & - & $\mathrm{X}$ & AUT & $\mathrm{C}$ & I & marrom-vináceo & drupa \\
\hline \multicolumn{10}{|l|}{ Rhamnaceae } \\
\hline Colubrina glandulosa Perkins* & DO & - & - & $\mathrm{X}$ & AUT & S & $\mathrm{D}$ & vináceo & cocarium \\
\hline Ziziphus joazeiro Mart. & DO & - & $\mathrm{X}$ & - & $\mathrm{ZOO}$ & $\mathrm{C}$ & I & amarelo & drupa \\
\hline
\end{tabular}


1044 Silva e Rodal: Padrões das síndromes de dispersão de plantas em áreas com diferentes graus de pluviosidade, PE, Brasil

Tabela 1 (Continuação).

\begin{tabular}{|c|c|c|c|c|c|c|c|c|c|}
\hline \multirow[b]{2}{*}{ Família / espécie } & \multicolumn{5}{|c|}{ Ocorrência } & \multirow[b]{2}{*}{$\mathrm{CO}$} & \multirow[b]{2}{*}{$\mathbf{D E}$} & \multirow[b]{2}{*}{ Coloração } & \multirow[b]{2}{*}{ Tipos de fruto } \\
\hline & $\mathbf{E V}$ & FLO & CAR & TAP & SD & & & & \\
\hline \multicolumn{10}{|l|}{ Rubiaceae } \\
\hline Alseis floribunda Schott & DO & - & - & $\mathrm{X}$ & ANE & $\mathrm{S}$ & $\mathrm{D}$ & marrom & cápsula \\
\hline Alseis pickelii Pilger \& Schmale & DO & - & - & $\mathrm{X}$ & ANE & $\mathrm{S}$ & $\mathrm{D}$ & marrom & cápsula \\
\hline Coutarea hexandra (Jacq.) K. Schum. & SB & - & $\mathrm{X}$ & - & ANE & $\mathrm{S}$ & $\mathrm{D}$ & marrom & cápsula \\
\hline Psychotria capitata Ruiz \& Pav. & SB & - & - & $\mathrm{X}$ & $\mathrm{ZOO}$ & $\mathrm{C}$ & I & verde & drupa \\
\hline Psychotria sp. & SB & - & - & $\mathrm{X}$ & $\mathrm{ZOO}$ & $\mathrm{C}$ & I & verde & baga \\
\hline \multicolumn{10}{|l|}{ Rutaceae } \\
\hline Metrodorea nigra A.St.Hil.* & SB & - & - & $\mathrm{X}$ & AUT & $\mathrm{S}$ & $\mathrm{D}$ & castanho & cocarium \\
\hline Zanthoxylum monogynum A.St.Hil.* & SB & - & - & $\mathrm{X}$ & AUT & $\mathrm{S}$ & $\mathrm{D}$ & castanho & cocarium \\
\hline \multicolumn{10}{|l|}{ Sapindaceae } \\
\hline Allophylus edulis (A. St.-Hil., Cambess. \& A. Juss.) Radlk. & SB & - & - & $\mathrm{X}$ & $\mathrm{ZOO}$ & $\mathrm{C}$ & I & vermelho & drupa \\
\hline Allophylus quercifolius Radlk. & SB & - & $\mathrm{X}$ & - & $\mathrm{ZOO}$ & $\mathrm{C}$ & I & amarelo & drupa \\
\hline Cupania oblongifolia Mart.* & DO & - & - & $\mathrm{X}$ & $\mathrm{ZOO}$ & $\mathrm{C}$ & $\mathrm{D}$ & marrom & cápsula \\
\hline Cupania racemosa (Vell.) Radlk. & DO & - & - & $\mathrm{X}$ & $\mathrm{ZOO}$ & $\mathrm{C}$ & $\mathrm{D}$ & verde & cápsula \\
\hline Cupania revoluta Radlk.* & DO & - & - & $\mathrm{X}$ & $\mathrm{ZOO}$ & $\mathrm{C}$ & $\mathrm{D}$ & marrom & cápsula \\
\hline \multicolumn{10}{|l|}{ Sapotaceae } \\
\hline Pouteria glomerata (Miq.) Radlk. & DO & - & - & $\mathrm{X}$ & $\mathrm{ZOO}$ & $\mathrm{C}$ & I & amarelo & drupa \\
\hline Pradosia verrucosa Ducke & DO & - & - & $\mathrm{X}$ & $\mathrm{ZOO}$ & $\mathrm{C}$ & I & amarelo & drupa \\
\hline Sapotaceae A & SB & - & - & $\mathrm{X}$ & $\mathrm{ZOO}$ & $\mathrm{C}$ & I & amarelo & baga \\
\hline \multicolumn{10}{|l|}{ Solanaceae } \\
\hline Solanum sp. & SB & - & - & $\mathrm{X}$ & $\mathrm{ZOO}$ & $\mathrm{C}$ & I & verde & baga \\
\hline Solanaceae A & SB & - & - & $\mathrm{X}$ & $\mathrm{ZOO}$ & $\mathrm{C}$ & I & verde & baga \\
\hline \multicolumn{10}{|l|}{ Sterculiaceae } \\
\hline Helicteris macropetala St. Hil.* & SB & - & $\mathrm{X}$ & - & AUT & $\mathrm{S}$ & $\mathrm{D}$ & negra & cápsula \\
\hline \multicolumn{10}{|l|}{ Tiliaceae } \\
\hline Apeiba sp. & DO & - & - & $\mathrm{X}$ & AUT & $\mathrm{S}$ & I & negra & baga \\
\hline Christiana africana DC.* & DO & - & - & $\mathrm{X}$ & AUT & $\mathrm{S}$ & $\mathrm{D}$ & verde & folículo \\
\hline Luehea paniculata Mart.* & DO & - & - & $\mathrm{X}$ & ANE & $\mathrm{S}$ & $\mathrm{D}$ & verde & folículo \\
\hline \multicolumn{10}{|l|}{ Verbenaceae } \\
\hline Lantana camara $\mathrm{L}$. & SB & - & $\mathrm{X}$ & - & $\mathrm{ZOO}$ & $\mathrm{C}$ & $\mathrm{I}$ & azul & drupa \\
\hline
\end{tabular}

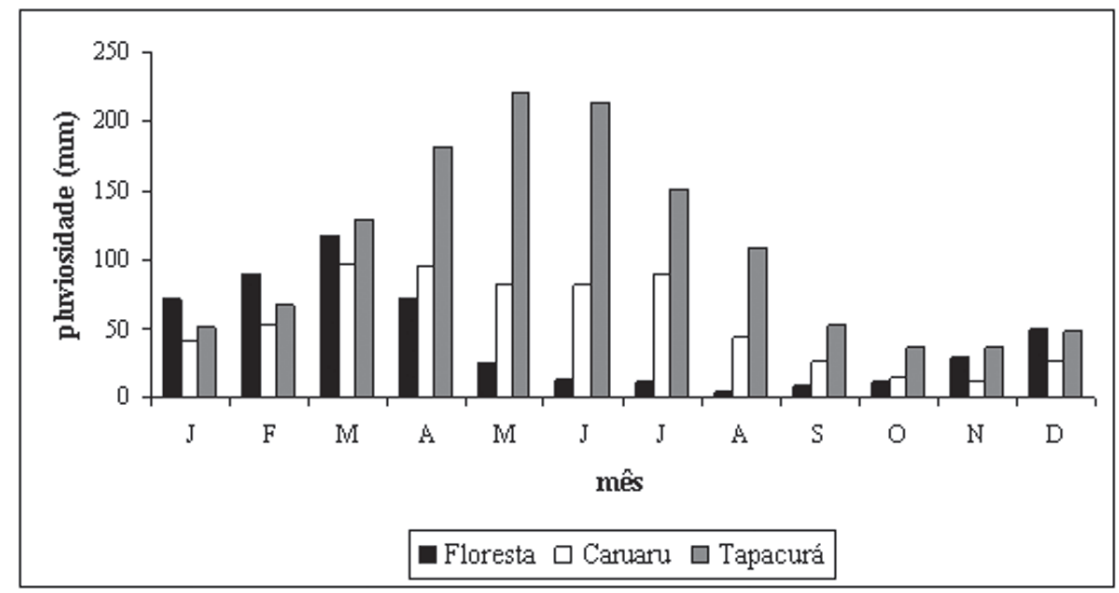

Figura 1. Precipitação média anual (mm) dos municípios de Floresta, Caruaru e Tapacurá, PE, Brasil.

especialmente pela presença de representantes da família Myrtaceae (Fig. 3). Visto que a anemocoria é comumente representada no dossel de áreas mais secas, à exceção de Coutarea hexandra, todas as espécies dispersadas pelo vento ocorreram no dossel.
Os resultados das análises de correlação de Spearman entre precipitação e síndromes de dispersão no dossel e sub-bosque das três áreas aqui estudadas e encontradas nos trabalhos de Machado et al. (1997) e Griz \& Machado (2001) mostraram correlação positiva e significativa entre zoocoria e 


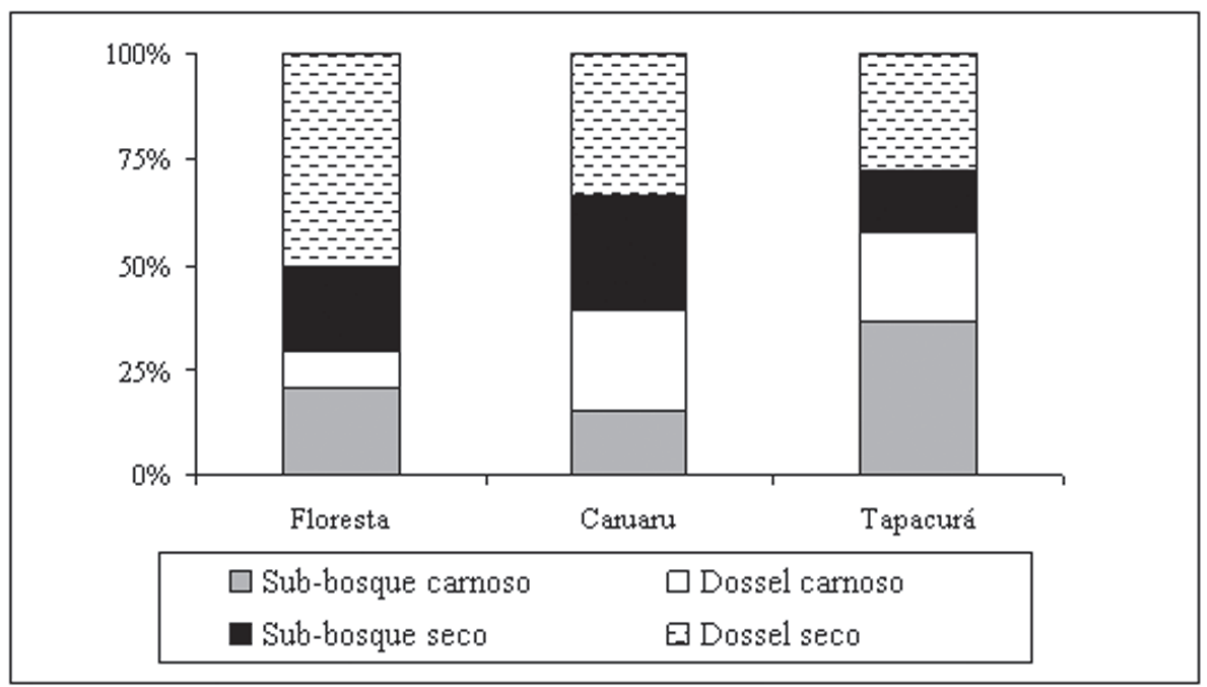

Figura 2. Porcentagem de espécies com frutos secos e carnosos no dossel e sub-bosque em Floresta, Caruaru e Tapacurá, PE, Brasil.

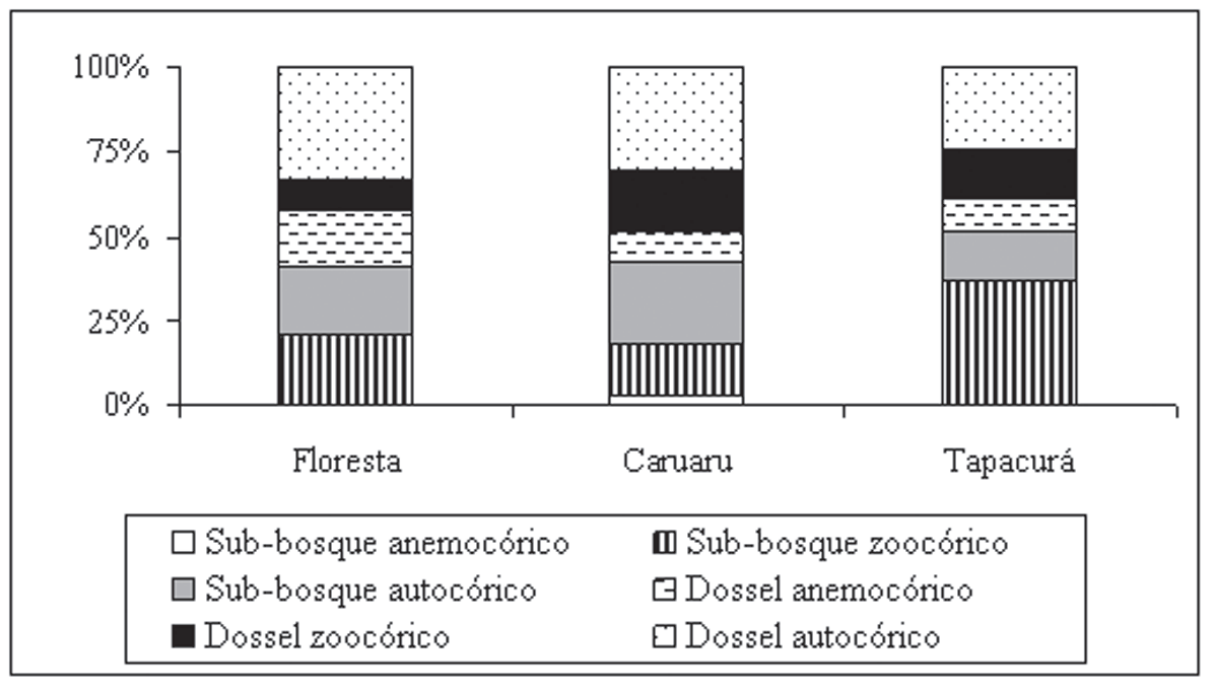

Figura 3. Porcentagem de espécies com frutos autocóricos, zoocóricos e anemocóricos no dossel e sub-bosque em Floresta, Caruaru e Tapacurá, PE, Brasil.

o sub-bosque da floresta estacional de Tapacurá $(\mathrm{p}=0,0374)$ e na proporção da anemocoria no dossel da caatinga estudada por Machado et al. $(1997)(\mathrm{p}=0,0374)$.

\section{Discussão}

Os resultados das proporções das síndromes mostraram que, nas áreas de estudo com precipitação anual inferior a 700 mm (Floresta e Caruaru), houve predomínio de vetores abióticos, enquanto que na floresta estacional de Tapacurá, mais de $50 \%$ das espécies apresentaram vetores bióticos, confirmando a literatura, que relata a predominância de vetores abióticos em áreas secas e de bióticos em áreas úmidas (Frankie et al. 1974, Howe \& Smallwood 1982, Gentry 1983, Butler et al. 2007).

Os poucos trabalhos que estudaram as síndromes de dispersão nas caatingas de Pernambuco também indicaram o predomínio de vetores abióticos (Machado et al. 1997, Griz \& Machado 2001, para revisão ver Barbosa et al. 2002). Corroborando os padrões relatados na literatura, as análises de correlação de Spearman entre precipitação e as síndromes de dispersão nos estratos da vegetação (dossel e sub-bosque) mostraram a importância da anemocoria no dossel da caatinga estudada por Machado et al. (1997) e da zoocoria no sub-bosque da floresta estacional de Tapacurá. Cabe destacar que a realização de mais estudos englobando diferentes tipos vegetacionais que ocorram ao longo do gradiente pluviométrico no estado de Pernambuco permitirá maiores considerações.

Sabe-se que espécies que apresentam diásporos adaptados para dispersão pelo vento geralmente apresentam maiores chances de ocupar ambientes mais abertos, sendo esperado que espécies anemocóricas predominem no estrato 
superior, ficando, eventualmente, sujeitas a ventos ocorrentes no dossel da floresta (Giehl et al. 2007, Yamamoto et al. 2007). A esse respeito, Nathan \& Katul (2005) observaram que o fluxo de vento, na maioria das vezes, pode ser afetado pela variação na folhagem do dossel, influenciando na dispersão a longa distância, característica de plantas anemocóricas. Essa variação é bem exemplificada em Floresta, Caruaru e Serra Talhada estudada por Machado et al. (1997), áreas onde a vegetação é caducifólia, facilitando a dispersão de espécies arbóreas como Myracrodruon urundeuva e Schinopsis brasiliensis durante a estação seca. Vale a pena destacar que Coutarea hexandra foi a única espécie dispersa pelo vento que ocorreu no sub-bosque. Segundo Justiniano \& Fredericksen (2000), o ambiente de sub-bosque não parece favorecer diásporos anemocóricos, já que nele a velocidade do vento é menor.

Apesar de Giehl et al. (2007) observarem que é comum a presença de espécies zoocóricas em todos os estratos florestais, de acordo com Mikich \& Silva (2001), apenas animais que apresentam facilidade de deslocamento entre os estratos podem tirar amplo proveito da disponibilidade de recursos alimentares. Na floresta estacional estudada (Tapacurá), mais de 70\% das espécies zoocóricas ocorrem no sub-bosque, onde há o predomínio de Myrtaceae (9 espécies), família caracterizada por apresentar frutos carnosos bacáceos, que são comumente dispersos por aves, macacos, roedores, morcegos (Barroso et al. 1999). Padrão semelhante foi relatado por Wikander (1984) e Justiniano \& Fredericksen (2000) em florestas secas na Venezuela e Bolívia, respectivamente, onde registraram maior número de espécies zoocóricas no sub-bosque.

Finalmente, pode-se concluir que, associados à diminuição da pluviosidade, os vetores abióticos tornam-se mais importantes em áreas de caatinga, vegetação não florestal caducifólia espinhosa característica do nordeste semi-árido brasileiro, e que as proporções das síndromes de dispersão variam de acordo com a estratificação, sendo a autocoria e a anemocoria mais representativas nas áreas mais secas, como Floresta e Caruaru, e havendo maior proporção de zoocoria no sub-bosque de florestas estacionais, como Tapacurá.

\section{Agradecimentos}

Ao Conselho Nacional de Pesquisa e à Fundação de Amparo à Ciência e Tecnologia pelo apoio financeiro concedido à primeira autora e pela bolsa de produtividade da segunda autora.

\section{Referências bibliográficas}

Alcoforado-Filho, F.G.; Sampaio, E.V.S.B. \& Rodal, M.J.N. 2003. Florística e fitossociologia de um remanescente de vegetação caducifólia espinhosa arbórea em Caruaru, Pernambuco. Acta Botanica Brasilica 17(2): 287-303.

Andrade, K.V.S.A. \& Rodal, M.J.N. 2004. Fisionomia e estrutura em um remanescente de floresta estacional semi-decidual de terras baixas no nordeste do Brasil. Revista Brasileira de Botânica 27(3): 463-474.

Ayres, M.; Ayres Jr.; M., Ayres, D.L. \& Santos A.S. 2000. Bioestat 2.0: Aplicações estatísticas nas áreas das ciências biológicas e médicas. Sociedade Civil Mamirauá/ MCT-CNPq, Belém, pp.259.
Barbosa, D.C.A., Hamburgo-Alves, J.L., Prazeres, S.M. \& Paiva, A.M.A. 1989. Dados fenológicos de 10 espécies arbóreas de uma área de Caatinga (Alagoinha - PE). Acta Botanica Brasilica 3: 109-117.

Barbosa, D.C.A., Silva, P.G.G. \& Barbosa, M.C.A. 2002. Tipos de frutos e síndromes de dispersão de espécies lenhosas da caatinga de Pernambuco. Pp. 609-621. In: M.Tabarelli \& J.M.C. Silva (eds.) Diagnóstico da Biodiversidade de Pernambuco, vol.2. SECTMA e Editora Massangana, Recife.

Barroso, G.M., Morim, M.P., Peixoto, A.L. \& Ichaso, C.L.F. 1999. Frutos e sementes: Morfologia aplicada à sistemática de Dicotiledôneas. Editora da Universidade Federal de Viçosa. Viçosa, pp. 443.

Butler, D.W., Green, R.J., Lamb, D., McDonald, W.J.F. \& Forster, P.I. 2007. Biogeography of seed-dispersal syndromes, life-forms and seed sizes among woody rain-forest plants in Australia's subtropics. Journal of Biogeography 34(10): 1736-1750.

Costa, R.C. \& Araújo, F.S. 2003. Densidade, germinação e flora do banco de sementes no solo, no final da estação seca, em uma área de Caatinga, Quixadá, CE. Acta Botanica Brasilica 17(2): 259-264.

Cronquist, A. 1981. An integrated system of classification of flowering plants. Columbia University Press, New York, pp. 1262.

Drezner, T.D., Fall, P.L. \& Stromberg, J.C. 2001. Plant distribution and dispersal mechanisms at the Hassayampa River Preserve, Arizona, USA. Global Ecology \& Biogeography 10(2): 205-217.

Fleming, T.H., Breitwisch, R. \& Whitesides, G.H. 1987. Patterns of tropical vertebrate frugivore diversity. Annual Review of Ecology and Systematics 18: 91-109.Frankie, G.W., Baker, H.G. \& Opler, P.A. 1974. Comparative phenological studies of trees in tropical wet and dry forests in the lowlands of Costa Rica. Journal of Ecology 62(3): 881-913.

Gentry, A.H. 1983. Dispersal ecology and diversity in neotropical forest communities. Sonderband Naturwissenschaftlicher Verein Hamburg 7: 303-314.

Giehl, E.L.H., Athayde, E.A., Budke, J.C., Gesing, J.P.A., Einsiger, S.M. \& Canto-Dorow, T.S. 2007. Espectro e distribuição vertical das estratégias de dispersão de diásporos do componente arbóreo em uma floresta estacional no sul do Brasil. Acta Botanica Brasílica 21(1): 137-145.

Griz, L.M.S. \& Machado, I.C.S. 1998. Aspectos morfológicos e síndromes de dispersão de frutos e sementes na Reserva Ecológica de Dois Irmãos. Pp. 197-224. In: I. C.Machado, A. V. Lopes, \& K. C. Pôrto (orgs.). Reserva Ecológica de Dois Irmãos: Estudos em um Remanescente de Mata Atlântica em área urbana (Recife-Pernambuco-Brasil). Editora Universitária da Universidade Federal de Pernambuco, Recife.

Griz, L.M.S. \& Machado, I.C.S. 2001. Fruiting phenology and seed dispersal syndromes in caatinga, a tropical dry forest in the Northeast of Brazil. Journal of Tropical Ecology 17: 303-321.

Griz, L.M.S., Machado, I.C.S. \& Tabarelli, M. 2002. Ecologia de dispersão de sementes: progressos e perspectivas. Pp. 597-608. In: M. Tabarelli \& J. M. C. Silva (orgs.). Diagnóstico da Biodiversidade de Pernambuco. Secretaria de Ciência, Tecnologia e Meio Ambiente, Fundação Joaquim Nabuco e Editora Massangana. Recife. vol. 2.

Howe, H.F. \& Smallwood, J. 1982. Ecology of seed dispersal. Annual Review of Ecology and Systematics 13: 201-228.

Justiniano, M.J. \& Fredericksen, T.S. 2000. Phenology of tree species in Bolivian dry forests. Biotropica 32(2): 276-281.

LAMEPE. 2009. Disponível em: http://www.itep.com.br/LAMEPE.asp (acesso: 03.01.09).

Machado, I.C.S. \& Lopes, A.V. 2004. Floral traits and pollination systems in the caatinga, a Brazilian tropical dry forest. Annals of Botany 94(3): 365-376.

Machado, I.C.S., Barros, M. \& Sampaio, E.V.S.B. 1997. Phenology of caatinga species at Serra Talhada, PE, Northeastern Brazil. Biotropica 29(1): 57-68.

Mantovani, W \& Martins, F.R. 1988. Variações fenológicas das espécies do cerrado da Reserva Biológica de Moji Guaçu, Estado de São Paulo. Revista Brasileira de Botânica 11(2): 101-112.

Mikich, S.B. \& Silva, S.M. 2001. Composição florística e fenologia das espécies zoocóricas de remanescentes de floresta estacional semidecidual no centro-oeste do Paraná, Brasil. Acta Botanica Brasílica 15: 89-113. 
Mooney, H.A., Bullock, S.H. \& Medina, E. 1995. Introduction. In: S.H Bullock, H.A. Mooney \& E. Medina, (eds.). Seasonally dry forests Cambridge University Press. Cambridge. Pp. 1-8.

Morellato, P.C. \& Leitão-Filho, H.F. 1996. Reproductive phenology of climbers in a Southeastern Brazilian Forest. Biotropica 28 (2): 180191.

Nathan, R. \& Katul, G.G. 2005. Foliage shedding in deciduous forests lifts up long-distance seed dispersal by wind. Proceedings of the Nacional Academy of Sciences of the United States of America 102 (23): 8251-8256.

Pianka, E.R. 1982. Ecología evolutiva. Universidad de Texas. Austin. 365p.

Pohill, R.M., Raven, P.H. \& Serton, C.H. 1981. Evolution and systematics of the Leguminosae. Pp. 1-26.In: R.M. Pohill \& P.H. Haven (eds.) Advances in legume systematics, apart I. Kew: Royal Botanic Garden.

Rey, P.J., Garrido, J.L., Alcántara, J.M., Ramírez, J.M., Aguilera, A., García, L., Manzaneda, A.J. \& Fernández, R. 2002. Spatial variation in ant and rodent post-dispersal predation of vertebrate-dispersed seeds. Functional Ecology 16(6): 773-781.

Rodal, M.J.N., Costa, K.C.C. \& Lins-e-Silva, A.C.B. 2008 Estrutura da vegetação caducifólia espinhosa (Caatinga) de uma área do sertão central de Pernambuco. Hoehnea 35: 209-217.

Rodal. M.J.N \& Sales, M.F. 2007. Composição da flora vascular em um remanescente de floresta montana no semi-árido do nordeste do Brasil. Hoehnea 34(4): 433-446.

Spjut, R.W. 1994. A systematic treatment of fruit types. New York Botanical Garden. New York, pp. 181
Tabarelli, M., Silva, A.V. \& Barbosa, D.C.A. 2003. Variation of seed dispersal spectrum of woody plants across a rainfall gradient in northeastern Brazil. Journal of Arid Environments 53(2). 197-210.

Van der Pijl, L. 1982. Principles of dispersal in higher plants. Springer Verlag. New York. 212p.

Veloso, H.P., Rangel Filho, A.L.R. \& Lima, J.C.A. 1991. Classificação da vegetação brasileira, adaptada a um sistema universal. IBGE. Rio de Janeiro. 123p.

Vicente, A., Santos, A. M. M. \& Tabarelli, M. 2003. Variação no modo de dispersão de espécies lenhosas em um gradiente de precipitação entre floresta seca e úmida no Nordeste do Brasil. Pp.565-592. In: I.R. Leal, M. Tabarelli \& J.M.C. Silva (orgs.). Ecologia e Conservação da Caatinga. Editora Universitária da Universidade Federal de Pernambuco, Recife.

Weiser, V. L. \& Godoy, S. A. P. 2001. Florística em um hectare de cerrado stricto sensu na arie - Cerrado Pé-de-Gigante, Santa Rita do Passo Quarto, SP. Acta Botanica Brasilica 15: 201-212.

Wikander, T. 1984. Mecanismos de dispersión de diásporas de una selva decidua en Venezuela. Biotropica 16(4): 276-283.

Willson, M. F. \& Traveset, A. 2000. The ecology of seed dispersal. Pp. 85-110. In: M. Fenner (ed.), Seeds: the ecology of regeneration in plant communities. CABI, Wallingford, UK.,

Yamamoto, L.F., Kinoshita, L.S. \& Martins, F.R. 2007. Síndromes de polinização e de dispersão em fragmentos da Floresta Estacional Semidecídua Montana, SP, Brasil. Acta Botanica Brasilica 21(3): 553-573. 